AperTO - Archivio Istituzionale Open Access dell'Università di Torino

\title{
Nation-wide measure of variability in HCMV, EBV and BKV DNA quantification among centers involved in monitoring transplanted patients
}

\section{This is the author's manuscript}

Original Citation:

\section{Availability:}

This version is available http://hdl.handle.net/2318/1633753

since 2017-05-15T08:58:32Z

Published version:

DOI:10.1016/j.jcv.2016.07.001

Terms of use:

Open Access

Anyone can freely access the full text of works made available as "Open Access". Works made available under a Creative Commons license can be used according to the terms and conditions of said license. Use of all other works requires consent of the right holder (author or publisher) if not exempted from copyright protection by the applicable law. 
1 Nation-wide measure of variability in HCMV, EBV and BKV DNA quantification among

centres involved in monitoring transplanted patients

3

4

Isabella Abbate $^{\mathrm{a}, 1}$, Antonio Piralla ${ }^{\mathrm{b}, 1}$, Agata Calvario ${ }^{\mathrm{c}}$, Annapaola Callegaro ${ }^{\mathrm{d}}$, Cristina Giraldi, Giovanna Lunghi ${ }^{\mathrm{f}}$, William Gennari ${ }^{\mathrm{g}}$, Giuseppe Sodano ${ }^{\mathrm{h}}$, Paolo Ravanini ${ }^{\mathrm{i}}$, Pier Giulio Conaldi ${ }^{\mathrm{j}}$, Marialinda Vatteroni ${ }^{\mathrm{k}}$, Aurelia Gaeta ${ }^{1}$, Pierpaolo Paba ${ }^{\mathrm{m}}$, Rossana Cavallo ${ }^{\mathrm{n}}$, Fausto Baldanti ${ }^{\mathrm{b}, \mathrm{o},{ }^{*} \text {, }}$ Tiziana Lazzarotto ${ }^{\mathrm{p}}$ and the AMCLI - Infections in Transplant Working Group (GLaIT) ${ }^{2}$

\section{${ }^{a}$ Laboratorio di Virologia, INMI L. Spallanzani, Roma}

${ }^{\mathrm{b}}$ SS Virologia Molecolare, SC Microbiologia e Virologia, Fondazione IRCCS Policlinico San Matteo, Pavia

${ }^{c}$ UOC Microbiologia e Virologia, Policlinico di Bari, Bari

${ }^{\mathrm{d}}$ UO di Microbiologia e Virologia, Ospedali Riuniti, Bergamo

${ }^{\mathrm{e}} \mathrm{UO}$ di Microbiologia e Virologia, P.O. Annunziata-A.O., Cosenza

${ }_{\mathrm{f}}^{\mathrm{f}}$ aboratorio di Virologia, IRCCS Ospedale Maggiore Policlinico Mangiagalli e Regina Elena, Milano

${ }^{g}$ UO di Microbiologia e Virologia, Azienda Ospedaliero-Universitaria di Modena, Modena

${ }^{h}$ UOC Microbiologia e Virologia, AORN Azienda Ospedaliera dei Colli, Ospedali Monaldi-Cotugno-CTO, Napoli

${ }^{i}$ UO di Microbiologia e Virologia, Ospedale Maggiore della Carità, Novara

${ }^{\mathrm{j}}$ Laboratorio di Patologia Clinica, Microbiologia e Virologia - ISMETT, Palermo

${ }^{k} U O$ di Virologia, Azienda Ospedaliera Universitaria Pisana, Pisa

${ }^{1}$ UOC Virologia, Policlinico Umberto I Sapienza, Roma

${ }^{\mathrm{m}}$ UOC Virologia Molecolare, Policlinico Fondazione Tor Vergata, Roma

${ }^{\mathrm{n}} \mathrm{SC}$ Microbiologia e Virologia, AOU Città della Salute e della Scienza, Università di Torino

${ }^{\circ}$ Sezione di Microbiologia, Dipartimento di Scienze Clinico-Chirurgiche, Diagnostiche e Pediatriche, Università degli studi di Pavia, Pavia

${ }^{\mathrm{P}}$ UO di Microbiologia, DIMES, Policlinico S. Orsola Malpighi, Università di Bologna, Bologna

*Corresponding author at: Department of Clinical, Surgical, Diagnostic and Pediatric Sciences,

University of Pavia, Viale Brambilla 74, Pavia, 27100, Italy. Fax: +39 0382502599.

E-mail addresses: f.baldanti@smatteo.pv.it, fausto.baldanti@unipv.it (F. Baldanti).

${ }^{1}$ Contributed equally

${ }^{2}$ Other participants in the GLaIT group along with their affiliation are listed in Appendix A.

Word count abstract: 248 (250)

Word count text: $1,587(2,500)$ 
ABSTRACT

2 Background: Inter-laboratory variability in quantifying pathogens involved in viral disease after transplantation may have a great impact on patient care, especially when pre-empitive strategies are

4 used for prevention.

5 Objectives: The aim of this study was to analyze the variability in quantifying CMV, EBV and 6 BKV DNA among 15 virology laboratories of the Italian Infections in Transplant Working Group 7 (GLaIT) involved in monitoring transplanted patients. Study Design: Panels from international Quality Control programs for Molecular Diagnostics consensus values were measured.

Results: $100 \%$ specificity was obtained with all panels. A sensitivity of $100 \%$ was achieved for BKV-DNA EBV and BKV were used. Intra- and inter-laboratory variability, as well as, deviation from QCMD EBV and BKV evaluation. Three CMV samples, with concentrations below $3 \log _{10}$ copies/ml, were not detected by a few centers. Mean intra-laboratory variability (\% CV) was 1.6 for CMV plasma and 3.0 for CMV WB. Mean inter-laboratory variability (\% CV) was below $15 \%$ for all the tested panels. An higher inter-laboratory variability was observed for CMV WB with respect to CMV plasma $(3.0$ vs $1.6 \% \mathrm{CV})$. The percentiles $87.7 \%, 58.6 \%, 89.6 \%$ and $74.7 \%$ fell within $\pm 0.5 \log _{10}$ difference of the consensus values for CMV plasma, CMV WB, EBV and BKV panels, respectively.

Conclusions: An acceptable intra- and inter-laboratory variability was observed in this study, in comparison with international standards. However, further harmonization in viral genome quantification is reasonable expectation for the future.

Keywords: multicenter evaluation; standardization; transplantation; CMV-DNA; EBV-DNA;

(2)




\section{Background}

2 Cytomegalovirus (CMV) and Epstein-Barr virus (EBV) are major causes of post-transplant viral

3 disease both in solid organ and hematopoietic stem cell transplantation, while Polioma BK virus

4 reactivation, with virus-associated nephropathy, represents the most frequent cause of graft loss

5 after renal transplantation [1,2]. The reliability and accuracy of viral load determination are

6 therefore critical for the management of transplant patients. In fact, virological monitoring of

7 transplanted patients is based on standardized protocols for genome quantification in order to apply

8 clinical cut-offs in pre-emptive approaches for disease prevention [3-6]. However, measurements of

9 viral load performed with commercially available assays might differ significantly, particularly

10 according to the extraction method used, which is a source of variability with different clinical

11 specimens. Finally, there is a need for an inter-laboratory comparison of results and evaluation of

12 individual assays with standardized panels, particularly with collaborative multicentre networks.

\section{2. Objectives}

15 The aim of this study was to analyze the variability obtained among 15 Italian virology

16 laboratories, belonging to the Working Group for Transplantation (Gruppo di Lavoro Infezioni nel

17 Trapianto, GLaIT) of the Italian Association of Clinical Microbiologists (Associazione

18 Microbiologi Clinici Italiani, AMCLI). Therefore, panels from international Quality Control

19 programs for Molecular Diagnostics (QCMD, year 2012), specific for the detection and 20 quantification of CMV in plasma, CMV in whole blood (WB), EBV and BKV were used.

3. Study design

23 3.1. QCMD samples

24 The QCMD panels used in the study were: QCMD 2012 CMV plasma, QCMD 2012 CMV WB, 25 QCMD 2012 EBV and QCMD 2012 BKV-JCV. A number of samples with various amounts of the 
1 different viruses suspended in an appropriate matrix and negative controls were tested in each

2 panel. For a detailed description and composition of the panels see www.qcmd.org.

\subsection{Extraction and quantitative real-time PCR assays}

5 Each sample was tested by each of the laboratories using commercial and in house methods

6 adopted for routinely virologic monitoring of transplanted patients (Table 1). Nucleic acid 7 extraction was performed by the majority of the laboratories using automatic extraction with commercial with sometimes in-house modifications; Real-time PCR amplification was carried out

9 by all the laboratories with commercially available kits, with only one exception. Quantitative

10 results were expressed as $\log _{10}$ copies $/ \mathrm{ml}$ for all three viruses tested. For positive samples detected

11 below the lowest limit of quantification, when a detected number of copies was not available, an 12 arbitrary value of half of the lowest limit of quantification was used.

\subsection{Statistical analysis}

15 Intra- and inter-laboratory variability was calculated, as well as, variation with respect to the 16 QCMD consensus as the coefficient of variation $(\% \mathrm{CV})$. The Pearson correlation analysis and the 17 Bland-Altman analysis were performed to examine the level of agreement between the 15 18 laboratories' results and the QCMD samples. Results were considered to be quantitatively 19 discordant when the results of the Bland-Altman analysis were discordant by more than $\pm 0.5 \log _{10}$ 20 of the QCMD consensus values. Statistical analysis was performed using Graph Pad Prism 21 software, version 5.00.288.

\section{Results}

The results obtained by the different GLaIT laboratories were analyzed to obtain a description of intra- and inter-laboratory variability and a quantitative comparison with respect to the consensus values reported by the different QCMD panels. 
For each of the four panels tested (QCMD CMV plasma, CMV WB, EBV and BKV) no false

2 positive results were obtained by any of the GLaIT laboratories (specificity 100\%). A sensitivity of

$3100 \%$ was achieved with the EBV and BKV evaluations. Concerning the CMV plasma panel,

4 sample \#3 (2.24 $\log _{10}$ copies/ml) was not detected by $1 / 15(6.6 \%)$ centres and sample \#4 (2.08 $\log _{10}$

5 copies/ml) was not detected by $4 / 15(26.6 \%)$ centres. For the CMV WB panel only sample \#8 (2.58

$6 \log _{10}$ copies/ml) was not detected by $4 / 13(30.8 \%)$ centres. For the BKV/JCV panel, no cross

7 reactivity with the JCV virus was observed (6 samples) and all of the centres detected all the five 8 samples containing BKV. variation $(\mathrm{CV})$ was 1.6 for CMV plasma and 3.0 for CMV WB.

In Table 2, for each sample the mean, standard deviation (SD), CV (\%), median and range of $\log _{10}$ copies/ml are reported. The mean SD for CMV plasma, CMV WB, EBV and BKV were respectively $0.27,0.49,0.25$ and 0.37 . The mean \% $\mathrm{CV}$ for CMV plasma, CMV WB, EBV and than $3 \log _{10}$ copies/ml.

In order to compare the results obtained by the different GLaIT laboratories with those of the international quality control study, consensus values for each QCMD panel were extrapolated and used for comparison. Significant correlations were observed in CMV WB, CMV plasma, EBV and BKV panel results with the Spearman coefficient which ranged from 0.82 to 0.96 (data not showed). Bland-Altman plots were used to describe the $\log _{10}$ difference between the GLaIT laboratory results and the consensus values (Figure 1). According to previous reports [7-9], \pm 0.5 $\log _{10}$ was considered an acceptable variability. In CMV the plasma panel, 114/130 (87.7\%) of the determinations were within $\pm 0.5 \log _{10}$ difference, while in the CMV WB panel only $51 / 87$ (58.6\%) 
1 were within $\pm 0.5 \log _{10}$ difference. In the CMV plasma panel, the majority of the discordant results

$2(14 / 16,87.5 \%)$ were observed in samples with a $<3.0 \log _{10}$ DNA copies number (Figure 1A), while

3 in the CMV WB panel discordant results were observed for all sample concentrations (Figure 1B).

4 In the EBV panel (Figure 1C), 120/134 (89.6\%) of the measurements were within a $\pm 0.5 \log _{10}$

5 difference, with no evident differences among different sample concentrations. In the BKV panel

6 (Figure 1D), a total of 56/75 (74.7\%) determinations fell within $\pm 0.5 \log _{10}$ difference; and for the

7 EBV panel, no differences among the different sample concentrations were detected.

\section{Discussion}

Since significant inter-laboratory variability in quantifying CMV, EBV and BKV genomes may

11 impact the quality of transplanted patient care, especially when pre-empitive strategies are used for 12 prevention, initiatives aimed at harmonizing viral genome quantification among different 13 laboratories should always be encouraged. In fact, transplant centres collect patients from all 14 Italians regions while post-transplant monitoring may be carried out by local laboratories. To the 15 best of our knowledge, this is the first report which simultaneously measures variability in 16 quantifying $\mathrm{CMV}, \mathrm{EBV}$ and $\mathrm{BKV}$ DNA which represent the three major viral pathogens 17 responsible for disease in solid organ transplantation.

18 Mission of the GLaIT group is to improve standardization of diagnostic procedures for microbiological monitoring of solid organs and stem cell transplant recipients. Two different studies aimed to measure variability in CMV and EBV DNA quantification have already been performed

21 [9,10]. Concerning CMV, the present study, in contrast with the 2009 report [9], takes into account both CMV DNA quantification in plasma and WB. As for the former CMV study, no false positive samples were obtained and a sensitivity of $100 \%$ was obtained in samples with a DNA load greater than $3 \log _{10}$ copies/ml. Although in the past a variability of less than $1 \log _{10}$ was obtained only in samples with a viral load greater than $3.7 \log _{10}$ copies/ml, the results reported here ranged from 0.93 
1 below $3.0 \log _{10}$ copies/ml. A greater variability was observed for CMV in WB with respect to

2 plasma; this is in line with the more complex matrix represented by blood, where nucleic acid

3 extraction is more laborious. At the same time, the CMV blood panel results, although more

4 variable, display a greater linearity. Overall, the $\%$ accuracy measured fell within $\pm 0.5 \log _{10}$ and

5 ranged from $58.6 \%$ to $89.6 \%$. This accuracy measured in a multicentre study is considered

6 acceptable and is higher than those observed in a similar study including fewer centres $(n=4)$ [11].

7 It should be underlined that in the present CMV quantification analysis, in contrast with previous 8 studies, all of the laboratories, with only one exception, used a commercial real-time PCR method 9 and only three different real-time methods were used. These real-time methods were however 10 associated with a variety of different manual or automated commercial and in house modified 11 protocols for nucleic acid extraction. It is reasonable to suppose that much of the variability 12 observed among the different quantifications was associated with the extraction procedures, rather 13 than the PCR amplification. This was also the case for EBV and BKV DNA determinations, where 14 as for CMV DNA, no false positive results were obtained for any samples. In the present study, the 15 best results (lower variability) were obtained with the EBV DNA panel. However, no direct 16 comparison can be made with the previous EBV study [10] due to the different composition of the 17 panels used for the evaluation. For BKV DNA, no cross reactivity was observed for samples 18 positive for the other Poliomavirus (JCV), included in the same QCMD panel and, although this 19 represents the first study by our group on quantification of this virus, an acceptable level of 20 variability was achieved. International standards are available since 2011 for CMV DNA and 2012 21 for EBV DNA $[12,13]$; this represents an opportunity to improve harmonization in CMV and EBV 22 genome quantification.

23 In conclusion, the results of this multicentre study indicate that CMV, EBV and BKV DNAemia 24 are quantified with acceptable variability using a variety of extraction volumes and protocols with 25 different commercial and in-house molecular protocols. 
2 None declared.

\section{$4 \quad$ Funding}

5 This work was supported by the Ministero della Salute, Fondazione IRCCS Policlinico San Matteo

$6 \quad$ Ricerca Corrente grant 80207.

\section{Ethical approval}

9 None. All experiments were performed with samples made available by QCMD 2012.

\section{Acknowledgements}

12 We thank Daniela Sartori for careful preparation of the manuscript and Laurene Kelly for English 13 revision. Appendix A. AMCLI-Infections in the Transplant Working Group (GLaIT) - list of other participants.

18 Maria Rosaria Capobianchi (Laboratorio di Virologia, INMI L. Spallanzani, Roma).

19 Maria Luisa Scarasciulli (Laboratorio di Virologia, U.O.C. Microbiologia e Virologia, Policlinico 20 di Bari, Bari).

21 Francesca Greco (UO di Microbiologia e Virologia, P.O. Annunziata-A.O., Cosenza).

22 Erminio Torresani (Laboratorio di Virologia, IRCCS Ospedale Maggiore Policlinico Mangiagalli 23 e Regina Elena, Milano).

24 Fabio Rumpianesi (UO di Microbiologia e Virologia, Azienda Ospedaliero-Universitaria di 25 Modena, Modena). 
1 Daniele Di Carlo (Laboratorio di Patologia Clinica, Microbiologia e Virologia - ISMETT, 2 Palermo).

3 Carlo Federico Perno (UOC Virologia Molecolare, Policlinico Fondazione Tor Vergata, Roma).

4 Cristina Costa (SC Microbiologia e Virologia, A.O. Città della Salute e della Scienza, Università 5 di Torino).

6 Liliana Gabrielli, Giulia Piccirilli (UO di Microbiologia, Policlinico S. Orsola Malpighi, 7 Bologna). 


\section{References}

2 [1] J.A. Fishman. infection in solid-organ transplant recipients. N. Engl. J. Med. 357 (2007) 26012614.

[2] H. H. Hirsch, P. Randhawa and the AST Infectious Diseases Community of Practice. BK Polyomavirus in solid organ transplantation. Am. J. Transplant. 13(S4) (2013) 179-188.

[3] R. Raymund. R.R. Razonable, R.T. Hayden. Clinical utility of viral load in management of cytomegalovirus infection after solid organ transplantation. Clin. Microbiol. Rev. 26 (2013) 703-727.

[4] M.L. Gulley and W. Tang. Using Epstein-Barr viral load assays to diagnose, monitor, and prevent posttransplant lymphoproliferative disorder. Clin. Microbiol. Rev. 23 (2010) 350-366.

[5] B.H. Chung, Y.A. Hong, H.G. Kim, I.O. Sun, S.R. Choi, H.S. Park, S.H. Lee, B.S. Choi, C.W. Park, Y.J. Choi, Y.S. Kim, C.W. Yang. Clinical usefulness of BK virus plasma quantitative PCR to prevent BK virus associated nephropathy. Transpl. Int. 25 (2012) 687-695.

[6] S. Hassan, C. Mittal, S. Amer, F. Khalid, A. Patel, R. Delbusto, L. Samuel, G. Alangaden, M. Ramesh. Currently recommended BK virus (BKV) plasma viral load cutoff of $\geq 4 \log 10 / \mathrm{mL}$ underestimates the diagnosis of $\mathrm{BKV}$-associated nephropathy: a single transplant center experience. Transpl. Infect. Dis. 16 (2014) 55-60.

[7] X.L. Pang, J.D. Fox, J.M. Fenton, G.G. Miller, A.M. Caliendo, J.K. Preiksaitis for the American Society of Transplantation Infectious Diseases Community of Practice; Canadian Society of Transplantation. Interlaboratory comparison of cytomegalovirus viral load assays. Am. J. Transplant. 9 (2009) 258-268.

[8] J.K. Preiksaitis, X.L. Pang, J.D. Fox, J.M. Fenton, A.M. Caliendo, G.G. Miller for the American Society of Transplantation Infectious Diseases Community of Practice. Interlaboratory comparison of Epstein-Barr virus viral load assays. Am. J. Transplant. 9 (2009) 269-279. 
1 [9] D. Lilleri ,T. Lazzarotto, V. Ghisetti ,P. Ravanini,M.R. Capobianchi, F. Baldanti, G. Gerna SIV-AMCLI Transplant Surveillance Group. Multicenter quality control study for human cytomegalovirus DNAemia quantification. New Microbiol. 32 (2009) 245-253.

[10]I. Abbate, M. Zanchetta, M. Gatti, L. Gabrielli, S. Zanussi, M.G. Milia, T. Lazzarotto, R. Tedeschi, V. Ghisetti, M. Clementi, A. De Rossi, F. Baldanti, M.R. Capobianchi. Multicenter comparative study of Epstein-Barr virus DNA quantification for virological monitoring in transplanted patients. J. Clin. Virol. 50 (2011) 224-229.

[11]J. Rychert, L. Danziger-Isakov, B. Yen-Lieberman, G. Storch, R. Buller, S.C. Sweet, A.K. Mehta, J.A. Cheeseman, P. Heeger, E.S. Rosenberg, J.A. Fishman. Multicenter comparison of laboratory performance in cytomegalovirus and Epstein-Barr virus viral load testing using international standards. Clin. Transplant. 28 (2014) 1416-1423.

[12]J.F. Fryer, A.B. Heath, R. Anderson, P.D. Minor and the collaborative study group. Collaborative study to evaluate the proposed $1^{\text {st }}$ WHO International Standard for human cytomegalovirus (HCMV) for nucleic acid amplification (NAT)-based assays. WHO ECBS Report (2010); WHO/BS/10.2138.

[13]J.F. Fryer, A.B. Heath, D.E. Wilkinson, P.D. Minor and the collaborative study group. Collaborative study to evaluate the proposed $1^{\text {st }}$ WHO International Standard for Epstein-Barr virus (EBV) for nucleic acid amplification (NAT)- based assays. WHO ECBS Report (2011); WHO/BS/11.2172.

[14]M. Furione, V. Rognoni, E. Cabano, F. Baldanti. Kinetics of human cytomegalovirus (HCMV) DNAemia in transplanted patients expressed in international units as determined with the Abbott RealTime CMV assay and an in-house assay. J. Clin. Virol. 55 (2012) 317-322.

[15]F. Baldanti, M. Gatti, M. Furione, S. Paolucci, C. Tinelli, P. Comoli, P. Merli, F. Locatelli. Kinetics of Epstein-Barr virus DNA load in different blood compartments of pediatric recipients of T-cell depleted HLA-haploidentical stem cell transplantation. J. Clin. Microbiol. 46 (2008) 3672-3677. 
1 [16]B.K. Saundh, S. Tibble, R. Baker, K. Sasnauskas, M. Harris, A. Hale. Different patterns of BK 2 and JC polyomavirus reactivation following renal transplantation. J. Clin. Pathol. 63 (2010) $3 \quad 714-718$.

4 
2 Figure 1. Bland-Altman plots are used to describe the Log difference between the GLaIT 3 laboratory results and QCMD consensus values. A (CMV plasma panel), B (CMV WB panel), $4 \quad \mathrm{C}(\mathrm{EBV}$ panel) and D (BKV panel).

5 
Table 1. Methods for viral DNA extraction and quantification.

\begin{tabular}{|c|c|c|c|c|c|c|c|}
\hline \multirow[t]{2}{*}{$\begin{array}{l}\text { Virus } \\
\text { target }\end{array}$} & \multirow[t]{2}{*}{$\begin{array}{c}\text { Center } \\
\#\end{array}$} & \multicolumn{2}{|l|}{ Nucleic acid extraction } & \multirow[t]{2}{*}{ Input volume $(\mu \mathrm{l})$} & \multirow[t]{2}{*}{ Output volume $(\mu \mathrm{I})$} & \multirow[t]{2}{*}{ Amplification method } & \multirow[t]{2}{*}{$\begin{array}{c}\text { Real-time PCR } \\
\text { instrument }\end{array}$} \\
\hline & & $\begin{array}{l}\text { Instrument } \\
\end{array}$ & Protocol & & & & \\
\hline \multirow[t]{17}{*}{$\overline{\mathrm{CMV}}$} & 1 & QIA Symphony & DSP virus/pathogen (modified) & 400 (200 for WB) & $90(90$ for $\mathrm{WB})$ & CMV Trender Affigene & Stratagene xp3000 \\
\hline & 2 & NucliSENS EasyMag & generic 2.0.1 & $250(100$ for $\mathrm{WB})$ & $25(25$ for $\mathrm{WB})$ & CMV Alert Real-Time, ELITechGroup & ABI Prism 7300 \\
\hline & 3 & QIA Symphony & DSP virus/pathogen (modified) & 1000 & 110 & CMV ELITe MGB Kit, ELITechGroup & ABI Prism 7300 \\
\hline & 4 & NucliSENS EasyMag & generic 2.0 .1 and specific 2.0 for WB & $500(200$ for $\mathrm{WB})$ & $55(55$ for $\mathrm{WB})$ & in house PCR (target US8)[14] & ABI Prism 7300 \\
\hline & 5 & NucliSENS EasyMag & generic 2.0.1 & 100 & 50 & CMV ELITe MGB Kit, ELITechGroup & ABI Prism 7300 \\
\hline & 6 & $\mathrm{x}$-tractor gene UV light & Helix DNA Corbet & 400 & 60 & CMV Alert Real-Time, ELITechGroup & ABI Prism 7300 \\
\hline & & & & 200 for WB & 150 for $\mathrm{WB}$ & CMV ELITe MGB Kit, ELITechGroup & \\
\hline & 7 & NucliSENS EasyMag & generic 2.0.1 & 500 & 50 & CMV ELITe MGB Kit, ELITechGroup & ABI Prism 7500 \\
\hline & & QIA Symphony & blood 200V6 for WB & 200 for WB & 200 for WB & & \\
\hline & 8 & NucliSENS EasyMag & generic 2.0.1 & $400(200$ for $\mathrm{WB})$ & 60 (85 for WB) & CMV Alert Real-Time, ELITechGroup & ABI Prism 7300 \\
\hline & 9 & NucliSENS EasyMag & generic 2.0.1 & $1000(200$ for $\mathrm{WB})$ & $25(55$ for $\mathrm{WB}$ & CMV Alert Real-Time, ELITechGroup & ABI Prism 7300 \\
\hline & 10 & QIA Symphony & DSP virus/pathogen and DSP DNA for WB & $500(200$ for $\mathrm{WB})$ & 90 (90 for WB) & CMV ELITe MGB Kit, ELITechGroup & ABI Prism 7300 \\
\hline & 11 & QIA Symphony & DSP virus/pathogen and DSP DNA for WB & 500 (200 for WB) & $140(90$ for $\mathrm{WB})$ & CMV ELITe MGB Kit, ELITechGroup & ABI Prism 7300 \\
\hline & 12 & NucliSENS EasyMag & specific 2.0 (modified for WB) & $500(100$ for $\mathrm{WB})$ & $100(50$ for $\mathrm{WB})$ & CMV ELITe MGB Kit, ELITechGroup & ABI Prism 7300 \\
\hline & 13 & NucliSENS EasyMag & generic 2.0.1 & $500(100$ for $\mathrm{WB})$ & $55(55$ for $\mathrm{WB})$ & CMV Alert Real-Time, ELITechGroup & ABI Prism 7300 \\
\hline & 14 & NucliSENS EasyMag & generic 2.0.1 & $500(200$ for WB $)$ & $55(55$ for $\mathrm{WB})$ & CMV Alert Real-Time, ELITechGroup & ABI Prism 7300 \\
\hline & 15 & Manual extraction & QIAamp blood mini kit & 200 & 100 & CMV r-gene Argene-Biomerieux & ABI Prism 7500 \\
\hline \multirow[t]{15}{*}{ EBV } & 1 & QIA Symphony & DSP virus/pathogen & 400 & 90 & EBV Trender Affigene & Stratagene $\mathrm{xp} 3000$ \\
\hline & 2 & NucliSENS EasyMag & generic 2.0.1 & 250 & 25 & EBV Alert Real-Time, ELITechgroup & ABI Prism 7300 \\
\hline & 3 & NucliSENS EasyMag & generic 2.0.1 & 500 & 55 & EBV ELITe MGB Kit, ELITechgroup & ABI Prism 7300 \\
\hline & 4 & NucliSENS EasyMag & generic 2.0.1 & 500 & 55 & in house PCR (target EBNA-1)[15] & ABI Prism 7300 \\
\hline & 5 & NucliSENS EasyMag & generic 2.0.1 & 100 & 100 & EBV ELITe MGB Kit, ELITechgroup & ABI Prism 7300 \\
\hline & 6 & $\mathrm{x}$-tractor gene UV light & Helix DNA Corbet & 400 & 60 & EBV Alert Real-Time, ELITechgroup & ABI Prism 7300 \\
\hline & 7 & NucliSENS EasyMag & generic 2.0.1 & 500 & 50 & EBV ELITe MGB Kit, ELITechgroup & ABI Prism 7500 \\
\hline & 8 & NucliSENS EasyMag & generic 2.0.1 & 400 & 60 & EBV Alert Real-Time, ELITechgroup & ABI Prism 7300 \\
\hline & 9 & NucliSENS EasyMag & generic 2.0.1 & 1000 & 25 & EBV Alert Real-Time, ELITechgroup & ABI Prism 7300 \\
\hline & 10 & NucliSENS EasyMag & generic 2.0.1 & 500 & 55 & EBV ELITe MGB Kit, ELITechgroup & ABI Prism 7300 \\
\hline & 11 & NucliSENS EasyMag & generic 2.0.1 & 1000 & 60 & EBV Alert Real-Time, ELITechgroup & ABI Prism 7300 \\
\hline & 12 & NucliSENS EasyMag & specific 2.0 & 500 & 100 & EBV ELITe MGB Kit, ELITechgroup & ABI Prism 7300 \\
\hline & 13 & NucliSENS EasyMag & generic 2.0.1 & 500 & 55 & EBV Alert Real-Time, ELITechgroup & ABI Prism 7300 \\
\hline & 14 & NucliSENS EasyMag & generic 2.0.1 & 500 & 55 & EBV Alert Real-Time, ELITechgroup & ABI Prism 7300 \\
\hline & 15 & Manual extraction & QIAamp blood mini kit & 200 & 100 & EBV R-gene Argene-Biomerieux & ABI Prism 7500 \\
\hline \multirow[t]{15}{*}{ BKV } & 1 & QIA Symphony & DSP virus/pathogen (modified) & 400 & 90 & BKV Trender Affigene & Stratagene xp3000 \\
\hline & 2 & NucliSENS EasyMag & generic 2.0.1 & 250 & 25 & BKV Alert Real-Time, ELITechgroup & ABI Prism 7300 \\
\hline & 3 & QIA Symphony & DSP virus/pathogen (modified) & 1000 & 110 & BKV ELITe MGB KIT, ELITechGroup & ABI Prism 7300 \\
\hline & 4 & NucliSENS EasyMag & generic 2.0.1 & 500 & 55 & in house PCR (target large T region)[16] & ABI Prism 7300 \\
\hline & 5 & NucliSENS EasyMag & generic 2.0.1 & 1000 & 100 & BKV ELITe MGB KIT, ELITechGroup & ABI Prism 7300 \\
\hline & 6 & $\mathrm{x}$-tractor gene UV light & Helix DNA Corbet & 400 & 60 & BKV ELITe MGB KIT, ELITechGroup & ABI Prism 7300 \\
\hline & 7 & NucliSENS EasyMag & generic 2.0.1 & 500 & 50 & BKV ELITe MGB KIT, ELITechGroup & ABI Prism 7500 \\
\hline & 8 & NucliSENS EasyMag & generic 2.0.1 & 400 & 60 & Light mix Polyomaviruses JC and BK (TibMolBiol) & Lightcycler 2.0 \\
\hline & 9 & NucliSENS EasyMag & generic 2.0.1 & 1000 & 25 & BKV Q-PCR Alert Kit, ELITechGroup & ABI Prism 7300 \\
\hline & 10 & NucliSENS EasyMag & generic 2.0.1 & 500 & 55 & BKV ELITe MGB KIT, ELITechGroup & ABI Prism 7300 \\
\hline & 11 & NucliSENS EasyMag & generic 2.0.1 & 1000 & 60 & BKV Alert Real-Time, ELITechgroup & ABI Prism 7300 \\
\hline & 12 & NucliSENS EasyMag & specific 2.0 & 500 & 100 & BKV ELITe MGB KIT, ELITechGroup & ABI Prism 7300 \\
\hline & 13 & NucliSENS EasyMag & generic 2.0.1 & 500 & 55 & BKV ELITe MGB KIT, ELITechGroup & ABI Prism 7300 \\
\hline & 14 & NucliSENS EasyMag & generic 2.0.1 & 500 & 55 & BKV ELITe MGB KIT, ELITechGroup & ABI Prism 7300 \\
\hline & 15 & Manual extraction & QIAamp blood mini kit & 200 & 100 & JC primers - probe + BKV R-gene, Argene-Biomerieux & ABI Prism 7500 \\
\hline
\end{tabular}


Table 2. Summary of quantitative performance for CMV plasma, CMV WB, EBV and BKV panels.

\begin{tabular}{llccccc}
\hline \multirow{2}{*}{ QCMV 2012 panel } & Sample & $\begin{array}{c}\text { No. of } \\
\text { values }\end{array}$ & $\begin{array}{c}\text { Mean } \pm \text { SD } \\
\text { (Log copies/ml) }\end{array}$ & $\begin{array}{c}\text { Inter-lab. CV } \\
(\%)\end{array}$ & $\begin{array}{c}\text { Median } \\
\text { (Log copies/ml) }\end{array}$ & $\begin{array}{c}\text { Range } \\
\text { (Log copies/ml) }\end{array}$ \\
\hline CMV plasma & CMV12-01 & 15 & $4.43 \pm 0.17$ & 3.93 & 4.43 & $4.12-4.65$ \\
& CMV12-02 & 15 & $3.82 \pm 0.29$ & 7.56 & 3.89 & $3.29-4.36$ \\
& CMV12-03 & 14 & $2.19 \pm 0.33$ & 12.65 & 2.17 & $1.56-2.53$ \\
& CMV12-04 & 11 & $2.05 \pm 0.46$ & 19.56 & 2.00 & $1.28-2.35$ \\
& CMV12-05 & 15 & $3.24 \pm 0.48$ & 14.84 & 3.40 & $2.04-3.66$ \\
& CMV12-06 & 15 & $3.43 \pm 0.21$ & 6.19 & 3.47 & $2.98-3.80$ \\
CMV WB & CMV12-07 & 15 & $3.46 \pm 0.18$ & 5.15 & 3.50 & $3.16-3.70$ \\
& CMV12-09 & 15 & $3.80 \pm 0.21$ & 5.60 & 3.83 & $3.29-4.14$ \\
& CMV12-10 & 15 & $2.74 \pm 0.26$ & 9.30 & 2.72 & $2.18-3.14$ \\
& CMV12-01 & 13 & $3.82 \pm 0.59$ & 15.33 & 3.78 & $2.78-4.56$ \\
CBV & CMV12-02 & 13 & $4.83 \pm 0.57$ & 11.90 & 4.96 & $3.89-5.50$ \\
& CMV12-04 & 13 & $3.01 \pm 0.43$ & 13.95 & 3.09 & $2.32-3.65$ \\
& CMV12-05 & 13 & $3.85 \pm 0.54$ & 14.06 & 3.82 & $3.15-4.51$ \\
& CMV12-07 & 13 & $4.03 \pm 0.41$ & 13.03 & 3.08 & $2.38-3.54$ \\
CMV12-08 & 9 & $2.63 \pm 0.50$ & 10.91 & 4.44 & $3.84-5.17$ \\
& EBV12-01 & 15 & $2.52 \pm 0.30$ & 16.57 & 2.73 & $1.81-3.05$ \\
& EBV12-02 & 15 & $3.57 \pm 0.24$ & 6.99 & 2.56 & $1.97-3.16$ \\
& EBV12-03 & 15 & $4.93 \pm 0.25$ & 5.12 & 3.59 & $3.22-4.20$ \\
EBV12-04 & 15 & $4.55 \pm 0.23$ & 5.17 & 4.87 & $4.59-5.53$ \\
& EBV12-05 & 15 & $4.12 \pm 0.23$ & 5.61 & 4.53 & $4.26-5.12$ \\
EBV12-06 & 15 & $4.26 \pm 0.21$ & 5.10 & 4.11 & $3.80-4.68$ \\
& EBV12-07 & 15 & $4.58 \pm 0.23$ & 5.19 & 4.20 & $3.98-4.77$ \\
EBV12-08 & 15 & $3.26 \pm 0.23$ & 7.02 & 4.49 & $4.32-5.21$ \\
& EBV12-09 & 15 & $3.09 \pm 0.26$ & 8.33 & 3.23 & $2.81-3.81$ \\
& BK12-02 & 15 & $3.58 \pm 0.38$ & 10.59 & 3.10 & $2.52-3.72$ \\
& BK12-03 & 15 & $2.43 \pm 0.34$ & 14.54 & 2.64 & $2.92-4.19$ \\
& BK12-07 & 15 & $1.72 \pm 0.48$ & 26.25 & 1.76 & $1.81-2.90$ \\
& BK12-08 & 15 & $4.64 \pm 0.39$ & 8.51 & 4.66 & $3.90-2.35$ \\
& BK12-12 & 15 & $5.14 \pm 0.34$ & 6.58 & 5.15 & $4.35-5.56$ \\
\hline
\end{tabular}

